\title{
Genesis
}

Manuscrits - Recherche - Invention

$40 \mid 2015$

Photo-graphies

\section{Abraham Constantin/Stendhal, Idées italiennes sur quelques tableaux célèbres, édition établie et présentée par Sandra Teroni et Hélène de Jacquelot, Paris, Beaux-Arts de Paris éditions, coll. « D'art en questions », 2013, 472 p.}

\section{Maria Teresa Giaveri}

\section{CpenEdition}

\section{Journals}

Édition électronique

URL : http://journals.openedition.org/genesis/1207

DOI : 10.4000/genesis. 1207

ISSN : 2268-1590

Éditeur :

Presses universitaires de Paris Sorbonne (PUPS), Société internationale de génétique artistique littéraire et scientifique (SIGALES)

Édition imprimée

Date de publication : 15 avril 2015

Pagination : 198-200

ISBN : 978-2-84050-992-9

ISSN : 1167-5101

\section{Référence électronique}

Maria Teresa Giaveri, « Abraham Constantin/Stendhal, Idées italiennes sur quelques tableaux célèbres, édition établie et présentée par Sandra Teroni et Hélène de Jacquelot, Paris, Beaux-Arts de Paris éditions, coll. «D'art en questions », 2013, 472 p. », Genesis [En ligne], 40 | 2015, mis en ligne le 11 décembre 2015, consulté le 22 septembre 2020. URL : http://journals.openedition.org/genesis/1207 DOI : https://doi.org/10.4000/genesis. 1207 
sage » à déchiffrer, de même que l'œuvre littéraire n'est pas réductible à un document de la période historique qui l'a vue naître. Le rapport entre texte et société est surtout pour lui un rapport de caractère linguistique et culturel, dont on doit cependant explorer les modalités toujours différentes.

Il est évident que ce travail herméneutique suppose une confiance fondamentale dans les capacités de l'interprète à surmonter la distance qui le sépare du texte, au moyen d'un ensemble de connaissances et d'une méthode de travail. Pour Segre, en effet, le passé est abordable, dans son extranéité même, et la tâche du critique est précisément celle, pour citer Gadamer, d'une « fusion des horizons ». C'est justement ici que le travail de l'interprète rejoint celui du philologue qui doit reconstruire un document abîmé par le temps. De ce point de vue, il n'y a pas de plus grande adhésion au texte que de la part de celui qui en suit le développement. «Le texte », écrit Segre, « se comprend véritablement quand on participe, en un certain sens, à son devenir, en parcourant les phases d'élaboration et en général de la tradition. C'est ce qui explique les études, développées au cours de ce siècle, de critique des variantes et de critique génétique » (p. XIV).

La partie « Dinamica delle varianti d'autore » (Dynamique des variantes d'auteur), la sixième de l'ouvrage (p. 651781 ), assume ainsi un relief central. Elle est introduite par l'article - rédigé en français pour la revue Genesis et paru dans le numéro 7 (1995) - « Critique des variantes et critique génétique ». Le point sur lequel Segre insiste, à la suite de Gianfranco Contini, est que le travail d'un auteur sur les variantes s'inscrit dans une logique de système. En effet, les variantes ne doivent pas être étudiées dans leur signification locale, comme des " améliorations » spécifiques d'un passage, mais comme une stratégie de la dynamique textuelle, c'està-dire, de sa transformation globale. Car chaque auteur tendrait, consciemment ou inconsciemment, à réaliser son propre
« système », comme on le déduit de l'essai «Sistema e strutture nelle "Soledades" di A. Machado » (Système et structures dans les «Soledades » d'A. Machado) (p. 690735), peut-être l'un des meilleurs de notre savant. C'est pour les mêmes raisons que Segre, dans sa pratique philologique, considère les variations du copiste non plus comme des erreurs ou des distractions, mais comme les symptômes d'un système linguistico-culturel qui se superpose sur celui de l'original, en créant des mélanges et des interférences. Ainsi la tâche du philologue est-elle d'historiciser les variantes de la tradition, ou plutôt de les ramener à leur code linguistico-culturel, sans cependant s'imaginer que son travail pourra vraiment conduire au rétablissement d'une « leçon originale » définitive, qui reste plus une orientation qu'un objectif effectivement atteint

Un autre point crucial de la méthode proposée par Segre concerne la recherche sur les sources (où le savant reprend et corrige la définition donnée par Kristeva du problème de l'intertextualité, en faisant appel à Bakhtine et en rapprochant de l'intertextualité la définition de l'interdiscursivité, p. 573-591), envisagée comme un problème herméneutique. En réalité pour Segre la connaissance des sources permet une interprétation différente et plus convaincante du texte, qui dévoile ainsi une partie de son sens. Dans ce cas aussi il s'agit d'une question de codes culturels, de superpositions et d'interférences.

Cependant, on n'aura pas compris ces explorations pénétrantes de problèmes théoriques et de textes, qui semblent ignorer les limites géographiques ou temporelles - sans jamais étaler le savoir et l'érudition qui néanmoins les sous-tendent -, si l'on n'a pas saisi au fond la grande valeur sociale que Segre reconnaît à la littérature. Ce n'est pas un hasard si le livre s'achève sur une partie qui comporte un seul essai, au titre emblématique, «Éthique et littérature ». Ici le savant cherche à répondre à l'éternelle question " qu'est-ce que la nature ? », en répondant à l'autre, tout aussi inévitable et fuyante, sur le «pour- quoi de la littérature ». Segre n'hésite pas à proposer un fondement éthique à cette raison d'être. Un principe qui ne se réduit pas à un moralisme idéologique, mais qui s'exprime plutôt par un sens des responsabilités envers son prochain. L'écrivain, écrit en effet Segre, est un «spécialiste de l'altérité ». Ce qui évidemment ne l'empêche pas d'exprimer dans son œuvre les contenus les plus divers, qui peuvent même aller jusqu'à la violence et la cruauté, sans que tout cela ne soit au détriment de la valeur esthétique. Et toutefois, « au lieu de poser le problème d'éthique et de littérature sous une forme théorique, le moment est peut-être venu de réclamer à la littérature cet intérêt éthique qui est essentiel non seulement pour notre intérêt littéraire mais pour la survie de la civilisation» (p. 1482).

Traduit de l'italien par Susan Wise

Abraham Constantin/Stendhal, Idées italiennes sur quelques tableaux célèbres, édition établie et présentée par Sandra Teroni et Hélène de Jacquelot, Paris, Beaux-Arts de Paris éditions, coll. « D'art en questions », 2013, 472 p.

\section{Compte rendu par Maria Teresa} Giaveri

« Dès le jour où [le] directeur du Cabinet Vieusseux, Alessandro Bonsanti, me mit sous les yeux la précieuse boîte des "épreuves" [des Idées italiennes...], me demandant ce que l'on pourrait faire pour leur valorisation, je n'eus qu'une idée : aller voir de près, ce que disent les manuscrits, prenant tout le temps qu'il faudrait. Quelque temps après, avec Hélène de Jacquelot, nous nous mîmes à la tâche, décidées à éclaircir le mystère. » Ainsi, dans les premières pages de son introduction critique, Sandra Teroni retrace le début de ce projet stendhalien qui a produit - après un long travail de recherche et d'analyse génétique - un très beau livre, publié par l'École nationale supérieure des beaux-arts de Paris, préfacé par François- 
René Martin et Pierre Wat et illustré non seulement par les images témoignant du processus d'écriture, mais aussi par les tableaux célèbres cités dans le titre.

$\mathrm{Au}$ commencement, il y a donc un « mystère », qui apparaît fascinant pour de multiples raisons.

En premier lieu, on s'interroge sur l'identité de l'auteur. Paru à Florence en 1840 sous le nom du peintre Abraham Constantin, le livre a été progressivement inclus dans l'œuvre de Stendhal après la découverte, en 1923, à la bibliothèque de Grenoble, du brouillon stendhalien d'un chapitre et, en 1924, d'un riche dossier (manuscrits, épreuves, lettres) au Cabinet Vieusseux de Florence. À partir de la deuxième édition des Euvres complètes de l'écrivain grenoblois (Paris, Le Divan, 1927-1937) jusqu'à la dernière édition proposée par le Cercle du Bibliophile (Genève, 1967-1974), on a vu se confirmer la thèse de Paul Arbelet - qui, en 1923, annonçait «Un livre inconnu de Stendhal » - et les études de Jahn Rusconi - qui en 1924 écrivait que « peu à peu la figure de Constantin se voile, s'éloigne, devant celle de Stendhal ». Mais aucune recherche approfondie n'ayant été menée sur le complexe avant-texte éparpillé dans plusieurs bibliothèques (Grenoble, Florence et, récemment, Genève), ce «nouveau livre » présenté aux lecteurs de Stendhal n'était que le volume publié sous le nom de Constantin en 1840, dont on avait changé l'indication auctoriale et auquel on avait ajouté un appareil de notes.

À juste titre, les deux spécialistes qui nous offrent maintenant cette édition exemplaire soulignent leur long travail : « Il s'agissait de repérer, classer, transcrire, interroger les pièces d'un parcours [...] dont la singularité et le charme résident dans la dissémination discrète d'indices contradictoires, dans le secret caché derrière un $j e$, dans le balancement entre symbiose et prise de distance » (p. 16).

La reconstitution de ce parcours d'écriture devient la reconstruction d'un rapport amical et d'une longue phase de la biographie de Stendhal; dès leur rencontre à Paris, en 1826, et surtout dès la lettre où Constantin annonce à ses parents, en 1831 : « J'attends un de mes amis qui est consul de France à Civita-Vecchia, lequel veut habiter Rome, il va arriver, je l'ai vu à mon passage, nous devons prendre un logement pour nous deux ce qui me sera fort agréable, car c'est un homme plein d'instruction et d'esprit », on peut suivre ponctuellement l'enchevêtrement des projets, des discussions, des voyages, des écritures des deux artistes.

Déjà célèbre pour ses peintures sur porcelaine, Abraham Constantin s'est rendu à Rome afin d'exécuter des copies des fresques de Raphaël pour la Manufacture de Sèvres ; son travail de peintre s'enrichit aussi d'un effort d'écriture, non seulement en rapport avec le livre sur les Idées italiennes, mais par la décision d'écrire son autobiographie (qu'il intitule $M a$ cinquantaine). En même temps, Stendhal alterne Cività-Vecchia et Rome, Paris et l'Italie, les conseils, les encouragements, les critiques à Constantin et des réflexions esthétiques au « but pédagogique » bien personnel. Les travaux de Sandra Teroni et d'Hélène de Jacquelot retracent ainsi les étapes de l'écriture des deux amis, dont la biographie nous a fait connaître l'entente «parfaite », mais dont les manuscrits, les lettres, les ajouts et les ratures sur les épreuves nous permettent de suivre les diverses curiosités intellectuelles et de mieux cerner leur personnalité respective. Le riche corpus de notes qui accompagne chaque chapitre (et qui offre aussi les images de nombreux documents), situe et explique en détail le processus complexe qui a donné naissance à cette œuvre sur la peinture italienne - et surtout sur la jouissance de la peinture.

Le «mystère » de l'identité de l'auteur apparaît ainsi attentivement décortiqué de page en page - et bien résumé et résolu dans les deux introductions critiques, où Sandra Teroni étudie «Une écriture à quatre mains » et Hélène de Jacquelot analyse « Constantin, Stendhal et Raphaël ». Mais « le secret caché derrière un je » se compose aussi d'autres éléments intéres- sants : l'aventure du texte s'enrichit très tôt d'une troisième figure, dont le rôle officiel d'éditeur masque une activité plus ponctuelle et généreuse, accomplie « avec intelligence, patience, amour, au-delà de ses fonctions » (p. 26). À coté du «personnage Stendhal » et de son ami Constantin, on peut en effet découvrir, dans ces pages, l'action culturelle et la personnalité de Giovan Pietro Vieusseux, fondateur du Cabinet de lecture florentin - un interlocuteur lucide et persévérant auquel l'enthousiasme de Constantin et l'intelligence multiforme de Stendhal avaient proposé une œuvre qui en vérité n'existait que sous forme de projet, et qui constituera progressivement, sous de multiples stimuli.

Le livre qui résulte de la recherche et de l'admirable travail critique de Sandra Teroni et d'Hélène de Jacquelot est presque un hypertexte : c'est un exemple de ce que seule peut accomplir une critique génétique qui met à contribution les instruments issus d'une sérieuse tradition philologique, tout en évitant ce « cimetière de variantes » détesté par quelques grands philologues italiens (et par tous les généticiens).

L'introduction de François-René Martin et de Pierre Wat souligne l'intérêt des Idées italiennes en ce qui concerne cet " art de voir », si cher à Stendhal : on retrouve, dans les descriptions des tableaux choisis, quelques idées proposées dans l'Histoire de la peinture en Italie ou dans les Promenades dans Rome. Loin des bibliothèques des érudits et du « savant Winckelmann », Stendhal y suggérait de «sentir par l'âme » la peinture et la sculpture de l'Italie : «Pour trouver du plaisir devant l'Apollon, il faut le regarder comme on suit un patineur rapide au bassin de la Villette. » Dans les Idées italiennes, cette éducation de l'œil se prolonge et trouve parfois des accents qui semblent appartenir aux «notes de chevet » de Sei Shonagon et à l'esthétique exquise de la culture japonaise de la période Heian : «La veille du jour où l'on doit quitter Rome revoir Michel-Ange et Raphaël, les yeux ont désappris le joli de 
Paris, et on les goûtera davantage [...]. Les Stanze se voient mieux lorsqu'il y a de la neige dans la cour, comme le 25 Mars $1840 »$ (p. 133).

Stendhal aime partager ce qu'il aime : tandis que Constantin (dont on peut lire, en annexe, la transcription du manuscrit initial) propose ses observations techniques, il voudrait éduquer à l'art - et à une perception artistique qui se veut italienne - ce nouveau public de « touristes », les héritiers bourgeois des aristocrates du Grand Tour du XVII et du XVIII ${ }^{\mathrm{e}}$ siècles. Autrefois, le voyage en Italie constituait une étape dans un parcours de formation culturelle qui était aussi un gage sociopolitique ; autrefois, les jeunes nobles voyageaient en compagnie de tutors et parfois de peintres et de littérateurs qui en traduisaient l'expérience en pages et en paysages ; autrefois, on lisait, au cours du voyage, des textes tels que le livre du prêtre catholique Richard Lassels (où on trouve, en 1670, la première attestation officielle de la formule "Grand Tour ») : The voyage of Italy: or, A compleat journey through Italy. In two parts. With the characters of the people, and the description of the chief towns, churches, monasteries, tombs, libraries, pallaces, villa's, gardens, pictures, statues, and antiquities. As also, of the interest, government, riches, force, \&c. of all the princes. With instructions concerning travel. By Richard Lassels, Gent. who travelled through Italy five times, as tutor to several of the English nobility and gentry.

C'est pour un nouveau public - loin de «nobility and gentry » - que Stendhal envisage ce nouveau Voyage of Italy: ses Idées italiennes sur quelques tableaux célèbres ne sont pas seulement un document constitutif de son esthétique, mais aussi un exemple de l'acuité de sa vision concernant cette transformation du « marché des biens symbolique » qui va caractériser le $\mathrm{XIX}^{\mathrm{e}}$ siècle. 